\title{
Surface generation and assessment for peripheral milling
}

\begin{abstract}
The rotating tool during peripheral milling filters disturbing vibrations between half of the rotation frequency and frequencies assigned to half the time necessary to generate a single cuttermark. This is a relatively large gap in the frequency range being not copied onto the finished surface. Roughness readings returning frequencies in that range are caused by other reasons like secondary fracture effects or the internal structure of the material. Superposition of vibrations at frequencies close to the rotation frequencies may cause interference patterns looking similar to the lines of cuttermarks but without representing the rotating frequency or the frequency of the disturbing vibration. This can also happen for perfectly aligned edges.
\end{abstract}

Keyword: Peripheral milling; Roughness; Cuttermarks; Vibration; Interference pattern 